\section{Regards sur l'économie allemande}

Bulletin économique du CIRAC

$110 \mid 2013$

Varia

\title{
Location : un marché sous tension dans les grandes villes allemandes
}

Solène Hazouard

\section{(2) OpenEdition}

\section{Journals}

Édition électronique

URL : http://journals.openedition.org/rea/4589

DOI : $10.4000 /$ rea.4589

ISSN : 1965-0787

Éditeur

CIRAC

\section{Édition imprimée}

Date de publication : 15 octobre 2013

Pagination : 19-26

ISSN : 1156-8992

\section{Référence électronique}

Solène Hazouard, "Location : un marché sous tension dans les grandes villes allemandes », Regards sur l'économie allemande [En ligne], 110 | octobre 2013, mis en ligne le 01 octobre 2015, consulté le 21 avril 2019. URL : http://journals.openedition.org/rea/4589; DOI : 10.4000/rea.4589 


\section{Location : un marché sous tension dans les grandes villes allemandes}

\section{Solène Hazouard}

En Allemagne, où le taux de locataires est particulièrement élevé en comparaison internationale (avec 46,6\% de la population selon les données 2011 EUSILC d'Eurostat), le thème de la hausse des loyers s'est invité dans la campagne électorale pour les législatives de 2013. Cette question est également d'actualité de ce côté-ci du Rhin, où 36,9\% des personnes vivaient en location en 2011 (soit 7,6 points de plus que la moyenne de l'Union européenne à 27). Le 12 septembre dernier, deux mesures de la loi pour l'accès au logement et un urbanisme rénové (Alur), portée par la ministre du Logement Cécile Duflot, ont ainsi été votées par l'Assemblée nationale : la garantie universelle des loyers et l'encadrement des loyers dans les zones où il existe un déséquilibre manifeste entre l'offre et la demande de logements.

Outre-Rhin, afin de remédier à la hausse du prix des loyers qui concerne actuellement une quinzaine de grandes villes allemandes, les pistes envisagées par les différents partis et experts portent pour leur part sur le plafonnement des loyers, la relance du logement social, l'augmentation des aides apportées aux locataires, la densification urbaine et le développement des communes environnantes.

\section{Un déséquilibre croissant entre offre et demande}

Les grandes villes allemandes deviennent de plus en plus attractives, aussi bien pour leurs habitants respectifs que pour les entreprises qui s'y implantent. Un cercle vertueux s'est ainsi créé : les jeunes diplômés, et notamment les femmes, sont en quête d'un lieu de vie leur permettant à la fois de trouver un emploi correspondant à leur niveau de qualification et de fonder une famille dans les meilleures conditions possibles, c'est-à-dire en disposant d'une offre de garde d'enfant adaptée à leur activité professionnelle. Les jeunes célibataires privilégient pour leur part un environnement urbain propice aux sorties. Réciproquement, les sociétés de services intensifs en savoir (banques, assurances et entreprises de conseil) choisissent prioritairement les villes dotées d'infrastructures de transport suprarégionales où se concentre une main-d'œuvre jeune et qualifiée. Rien d'étonnant dès lors à ce que, entre 2005 et 2011, la population ait augmenté de $9,4 \%$ à Munich, de 7,7 \% à Münster et à Potsdam, de $7 \%$ à Dresde, de $6,1 \%$ à Fribourg et à Francfort ou encore de $6 \%$ à Darmstadt.

Ce phénomène de migration accrue vers les villes, qui résulte de l'évolution des qualifications et de la tertiarisation de l'économie, va de pair avec l'éclatement du modèle familial. Entre 1991 et 2011, la taille moyenne des foyers s'est réduite de 2,27 à 2,02 personnes. Désormais, $20 \%$ des individus vivent seuls outre-Rhin, cette proportion atteignant même $30 \%$ dans les villes de plus de 500000 habitants, selon un sondage mené cette année par l'Institut für Demoskopie d'Allensbach (IfD) auprès d'un échantillon représentatif de 1500 personnes de 16 ans et plus. Conséquence directe de cette tendance (qui affecte également la France, avec 18,3 \% de solos en 2010 selon l'Insee) : de 2002 à 2012, le nombre de mé-

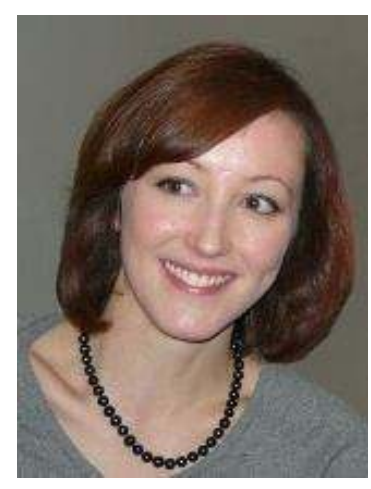

Solène Hazouard, Ingénieur d'études au CIRAC
Une demande stimulée par l'attrait croissant des grandes villes auprès des jeunes diplômés...
... et l'explosion du nombre de ménages 
Recul de la construction de logements après le boom de la réunification

L'offre ne répond plus à la demande dans les grandes villes allemandes

Un problème à la fois d'ordre quantitatif et qualitatif

Forte hausse des loyers à la relocation dans les métropoles

Le taux d'effort élevé en matière de logement... nages allemands est passé de 38,7 millions à 40,3 millions. Selon l'Office fédéral des statistiques, cette augmentation est vouée à se poursuivre dans les années à venir, un pic devant être atteint en 2025 avec un 41,1 millions de ménages.

Face à l'accroissement de la demande, l'offre n'a pas suivi dans les grandes villes allemandes économiquement attractives et universitaires. Ces dernières années en effet, un nombre insuffisant de logements a été construit pour répondre aux besoins actuels dans les régions en croissance démographique. Cela s'explique par le boom de la construction qui a immédiatement suivi la réunification allemande, stimulé notamment par les aides fiscales accordées dans les Länder de l'ex-RDA. Les logements érigés en trop grand nombre ont généré une distorsion du marché, avec pour effet un accroissement du taux de vacance et une stagnation des loyers. Le recul des nouvelles constructions observé à partir de 1996 venait donc en quelque sorte assainir le marché.

Toutefois, à la fin des années 2000, la construction de nouveaux logements a reculé plus vite que la demande, de sorte que son niveau demeure, encore aujourd'hui, inférieur aux besoins actuels. Le marché a ainsi enregistré une baisse de $36 \%$ entre 2006 et 2009 (BMVBS, 2013). Cela s'explique notamment par la suppression, en 2006, de la prime d'accès à la propriété (Eigenheimzulage), créée dix ans plus tôt et destinée à favoriser l'accession à la propriété des catégories sociales moins aisées (voir REA 102/11). Depuis 2009 cependant, le nombre d'autorisations de constructions augmente progressivement, de sorte qu'en 2012, le niveau de 2005 était de nouveau atteint. Dans un contexte de crise de la zone euro, d'incertitude sur les marchés boursiers et de crainte de l'inflation, l'immobilier s'impose davantage comme une valeur refuge, d'autant que les taux d'intérêts sont historiquement bas. Néanmoins, il manquerait toujours 250000 logements dans les grandes villes, selon l'Union allemande des locataires (Deutscher Mieterbund e.V.). Le Cabinet de Consulting Empirica indique pour sa part un taux très faible de logements vacants susceptibles d'être loués à Munich $(0,6 \%)$ et à Hambourg $(0,7 \%)$.

Le problème est ainsi d'ordre quantitatif : entre 2000 et 2010, dans le « top 7 » des villes allemandes (Berlin, Düsseldorf, Francfort-sur-le-Main, Hambourg, Cologne, Munich et Stuttgart), la construction de logements était inférieure de plus de moitié à l'augmentation du nombre de ménages. Cette faiblesse au niveau de l'offre peut s'expliquer par l'accroissement des coûts de construction et de modernisation énergétique et par le renchérissement du prix des terrains, du fait de leur rareté dans les zones privilégiées à l'intérieur de la plupart des villes. En outre, le déséquilibre est qualitatif : les nouvelles constructions, qui excèdent bien souvent les $100 \mathrm{~m}^{2}$, ne correspondent pas aux besoins réels des solos, de plus en plus nombreux sur le marché.

\section{Une pénurie de logements abordables dans les grandes villes}

Cette situation de tension sur le marché de l'immobilier, combinée à l'évolution qualitative de l'offre et au renforcement des normes relatives aux bâtiments, conduit à une augmentation du prix des loyers dans des métropoles comme Berlin, Hambourg ou Munich, et des villes universitaires comme Fribourg ou Münster. Un phénomène de grande ampleur, sachant que près de la moitié de la population allemande se concentre dans les grandes villes et dans les agglomérations. Ces cinq dernières années, les loyers des nouvelles locations et des relocations ont ainsi augmenté de plus de $20 \%$ à Hambourg et Berlin, d'environ $14 \%$ à Munich et à Francfort et de près de $11 \%$ à Stuttgart. Sur l'année 2012 uniquement, la hausse se situait entre $3 \%$ et $4 \%$ dans la quasi-totalité des grandes villes, à l'exception de Hambourg (Spars/Heinze, 2013).

Mais ce n'est pas tant la hausse des loyers que la stagnation, voire le recul des salaires chez les personnes peu qualifiées et la hausse des coûts annexes au logement qui ont engendré une explosion des frais liés du logement pour cer- 
taines couches de la population. En 2011, la part du revenu net disponible des ménages consacrée au logement (incluant les coûts pour l'eau, l'énergie et le traitement des eaux usées, les frais de chauffage, les dépenses consacrées à l'entretien du logement, les intérêts du prêt immobilier pour les propriétaires ainsi que les frais d'assurance) se montait à $28,3 \%$ en moyenne. Elle atteignait $39,1 \%$ pour les personnes vivant seules et $38,7 \%$ pour les parents isolés. Parmi les ménages les plus modestes, le taux d'effort pouvait atteindre $50 \%$ et grimper à $57,1 \%$ pour les personnes vivant seules menacées de pauvreté ou $52,3 \%$ pour les parents isolés menacés de pauvreté. Ainsi, en Allemagne en 2011, près d'une personne sur cinq (19,8 \%) trouvait que le coût du logement venait considérablement grever son budget (Destatis, 2013). Le sondage de I'IfD évoqué plus haut fait pour sa part apparaître que $64 \%$ des habitants des villes de 500000 habitants et plus considèrent les loyers comme trop élevés, alors que sur l'ensemble du territoire allemand, ce taux n'est que de $38 \%$.

Par ailleurs, les quartiers embourgeoisés ( gentrifiés ») dans des villes comme Berlin et Hambourg ont subi une modernisation forcée répondant au degré d'exigence et aux capacités économiques des classes moyennes et des hauts revenus, de sorte que les ménages modestes ont été contraints de se déplacer (Faller, 2012). Les logements proposés sur le marché, trop spacieux et trop onéreux, sont de plus en plus inaccessibles pour les petits ménages modestes composés d'étudiants, de personnes seules à faibles revenus, de retraités percevant une petite pension ou d'allocataires de l'Arbeitslosengeld II (" Hartz IV »), prestation versée aux personnes sans emploi en capacité de travailler. Selon une étude récente de l'entreprise de conseil RegioKontext, réalisée pour le compte des fédérations du secteur de l'immobilier, du syndicat de la construction IG Bau et de I'Union allemande des locataires, les classes moyennes seraient également de plus en plus concernées par la difficulté à trouver un logement à un prix abordable dans les centres-villes. Dès lors, la suburbanisation de la population (survenant pour des raisons économiques) reste un phénomène actuel (Spars, 2012).

La flambée des prix serait néanmoins à relativiser selon Michael Voigtländer, chercheur à l'Institut der Deutschen Wirtschaft (IW, Cologne). II précise en effet que les loyers pratiqués dans les grandes métropoles allemandes demeurent inférieurs à ceux des villes comme Paris ou Londres. Cela est dû à la structure polycentrique de l'Allemagne, au sein de laquelle des agglomérations comme Berlin, Munich et Hambourg constituent des pôles qui entrent en concurrence les uns avec les autres.

\section{Pistes envisagées pour assainir le marché}

\section{Les propositions politiques relatives au plafonnement des loyers}

Face à l'augmentation des loyers et dans un contexte de campagne électorale pour les législatives, les responsables politiques chrétiens-démocrates (CDU) et libéraux (FDP) ont entrepris de modifier le droit en matière de location, de sorte que depuis mai 2013, les Länder peuvent décider de plafonner la hausse du prix des loyers pour les baux existants à $15 \%$ sur trois ans (contre $20 \%$ auparavant) dans les villes où une offre suffisante de biens locatifs à des conditions raisonnables est menacée. Au cours de la campagne, les sociaux-démocrates (SPD) ont soumis l'idée d'un plafonnement à $15 \%$ sur quatre ans. Ils se sont également prononcés en faveur d'une limitation des tarifs des nouveaux contrats de location à $10 \%$ au-dessus du loyer habituellement pratiqué dans la zone géographique considérée. Dans son programme électoral, la CDU a réagi en proposant de limiter la hausse des loyers relative aux nouveaux contrats de location, mesure à laquelle les libéraux (FDP) sont défavorables. Néanmoins, la CDU rejetait elle-même quelque temps plus tard une proposition analogue des Verts au Bundestag.
... constitue le terreau de la gentrification

Des loyers qui restent inférieurs à ceux de Paris ou Londres

La limitation de la hausse des loyers : un thème électoral... 
... susceptible d'enrayer la reprise amorcée en 2010
Ces propositions font directement écho à l'opinion. En effet, conscients que les pouvoirs publics sont confrontés au choix de freiner l'augmentation des loyers ou de construire de nouveaux logements, la plupart des Allemands (54\%) se prononcent en faveur d'une limitation du prix des loyers par l'Etat, tandis que $29 \%$ privilégient en priorité l'augmentation de la construction de logements neufs. Toutefois, dans les grandes villes, le taux de personnes qui optent pour le frein à l'augmentation des loyers est inférieur à la moyenne nationale, avec $50 \%$ (IfD Allensbach, 2013).

La limitation de la hausse des loyers appliquée aux nouveaux contrats de location viendrait en effet freiner les investisseurs dans leurs projets de construction, et ce alors que la reprise enclenchée en 2010 pourrait garantir de nombreux nouveaux logements dans les prochaines années. Les experts rappellent que ce sont justement les interventions politiques sur le marché de l'immobilier (notamment les aides fiscales accordées dans les Länder de l'est de l'Allemagne) qui ont conduit à une " construction effrénée » dans les années 1990, entraînant ensuite un tassement de l'activité (Spars/Heinze, 2013). Or, dans les conditions actuelles du marché, la reprise devrait se poursuivre durant les quatre prochaines années (Dorffmeister/Gluch, 2013).

Pour ne pas dissuader les investisseurs de construire, il faut en effet garantir la stabilité du ratio prix d'achat/coût du loyer : l'évolution annuelle moyenne des prix d'habitation à l'achat est divisée par l'évolution des nouveaux loyers moyens. Depuis 2007, ce ratio a évolué de manière différente dans les cinq villes les plus peuplées outre-Rhin : tandis que Berlin, Francfort et Cologne se sont très légèrement éloignées de la moyenne allemande vers le haut, à Munich et à Hambourg, le décrochement est plus net. Ces deux villes ne présentent toutefois pas le risque d'une bulle spéculative. Cette évolution peut s'expliquer par le fait qu'en raison de la bonne santé économique de ces villes, la prime de risque est particulièrement faible et le potentiel d'augmentation des loyers, particulièrement élevé. Par ailleurs, il convient de noter qu'en valeur réelle, les prix des logements à l'achat ont subi une baisse en Allemagne entre 1994 et 2008. Cependant, les chercheurs de l'IW prévoient qu'en cas de nouvelle augmentation, une masse critique devrait bientôt être atteinte, laquelle aboutirait à d'importantes corrections de prix, au moins dans certains quartiers. Bloquer les loyers reviendrait à creuser artificiellement l'écart entre prix d'achat et coût du loyer, et rendrait la construction moins attractive pour les investisseurs en termes de rendement, ce qui prolongerait la situation de pénurie de logements dont souffre la demande (Haas/Henger/Voigtländer, 2013). D'autant qu'en moyenne sur le territoire allemand, le locataire verse l'équivalent du prix du logement à son propriétaire sur 20 ans. Dans les villes où le prix des logements s'est décorrélé des loyers, le propriétaire devra patienter davantage : 25 ans à Hambourg et à Munich, voire 26 ans à Berlin (FAZ, 2013).

\section{Soutien de l'offre, notamment en matière de logements sociaux}

Réintroduction de l'amortissement dégressif pour inciter à la construction

Positionnement des différents partis politiques sur les logements sociaux
En contrepartie du plafonnement des loyers, la CDU a émis l'idée de porter dans un premier temps le taux d'amortissement de $2 \%$ à $4 \%$ pour les nouvelles constructions. Cette réintroduction de l'amortissement dégressif représenterait un allègement fiscal pour les acteurs de la branche. Elle se justifierait par des exigences accrues en matière énergétique et stimulerait l'investissement dans la construction, permettant in fine d'amortir la hausse des loyers.

Par ailleurs, la construction de logements sociaux est revenue au cœur des débats. Relevant de la compétence des Länder depuis la réforme du fédéralisme de 2006, le secteur continue cependant de bénéficier d'une compensation annuelle de l'Etat fédéral à hauteur de 518 millions $€$, dont le versement est prévu jusqu'en 2019 (BMVBS, 2013). Ce montant, complété par les Länder, atteignait ainsi 1 milliard $€$ en 2010. Lors de la campagne de 2013, la CDU, le FDP et le SPD ont exigé que la somme allouée par l'Etat fédéral soit véritablement utilisée à cette fin par les Länder. Pour les deux grands partis CDU et SPD, les 
communes doivent en outre céder aux investisseurs les terrains qui leur appartiennent à des conditions favorables, lorsque ceux-ci réservent une partie des logements aux personnes socialement défavorisées. Les Verts préconisent pour leur part un soutien accru de l'Etat fédéral aux Länder et aux communes en matière de création de logements sociaux dans les limites géographiques des villes, de sorte qu'aucun ghetto ne soit constitué dans les banlieues et que soit ainsi préservée la mixité sociale dont font montre, encore aujourd'hui, les grandes villes allemandes.

Après la Seconde Guerre mondiale, plus de la moitié des nouvelles constructions ont bénéficié de subventions, permettant alors à de larges pans de la population de trouver un toit. Au début des années 1970, ce processus fut décrié, puis progressivement laissé à l'abandon car il avait généré des ghettos suburbains. Tandis qu'en 1990, l'Allemagne recensait encore 3,6 millions de logements sociaux à loyer modéré, ce chiffre est tombé à 1,6 million. Deux facteurs expliquent ce recul annuel de 100000 logements: l'arrivée du terme de la période de subventionnement et le recul du volume des aides accordées. Avec ce système, les investisseurs se voyaient rembourser une part importante de leurs frais d'investissement et de gestion, la plupart du temps sur plus de 30 ans. Le loyer calculé sur la base des coûts (Kostenmiete) était souvent situé audessus du niveau de celui du marché. Cela a coûté très cher aux villes.

Malgré ces mauvaises expériences passées, et devant l'écart grandissant entre l'offre et la demande, les grandes villes allemandes ont relancé la construction de logements sociaux. Dans une étude réalisée pour le compte des acteurs du secteur, l'Institut Pestel (centre de recherche créé en 1975 à l'initiative du cofondateur du Club de Rome Eduard Pestel) estime en effet actuellement à 5,6 millions le nombre de ménages potentiellement éligibles à un logement à loyer modéré (Pestel Institut, 2012). Tandis qu'à Cologne et à Munich, les programmes visant à construire de nouveaux logements sociaux sont mis en œuvre lorsque les moyens alloués par les Länder sont épuisés, à Francfort et à Stuttgart, les financements octroyés par le Land sont complétés par des fonds communaux.

Le modèle munichois sert de référence pour de nombreuses communes: l'octroi d'autorisations de construction ou de prêts à taux avantageux y va de pair avec le fait que les investisseurs exploitent eux-mêmes les zones d'habitation et consacrent $30 \%$ des terrains au logement social. Mais ce modèle a ses failles : le marché foncier y est tellement disputé que la part sociale induit une forte hausse sur le marché privé, qui devient ainsi hors de prix pour la classe moyenne. Selon les experts, le concept serait donc plus à même de fonctionner dans d'autres villes moins densément peuplées.

La ville-Etat de Hambourg, qui compte seulement $24,1 \%$ de propriétaires, a pour ambition de construire 6000 logements par an, parmi lesquels 2000 logements sociaux. Le coût annuel du projet « Vertrag für Hamburg - Wohnungsneubau », conclu en 2011 entre le Sénat de Hambourg et les districts (Bezirke) se monte à près de 100 millions $€$. II place les districts dans l'obligation d'établir des programmes de construction de logements neufs et de veiller à accélérer les procédures d'autorisation, qui ne doivent pas excéder six mois à compter de la réception de la demande. Outre les ménages à faibles revenus, les classes moyennes sont prises en considération : il leur sera réservé chaque année plus d'un tiers des 2000 logements sociaux prévus.

A Berlin, où le taux de propriétaires est le plus faible d'Allemagne (15,6 \%), il est question pour le Land de mettre à disposition des terrains sur lesquels un tiers des logements construits seront à loyer modéré. Ces logements pourraient ensuite être achetés et mis en location par des entreprises communales, la part restante étant gérée par des investisseurs. Sur ce sujet, les experts précisent la nécessité pour les villes de conserver des sociétés immobilières communales, bien souvent cédées au secteur privé par le passé (Spars/Heinze, 2013).
Le recul des logements à loyer modéré outre-Rhin...

... est désormais enrayé. Un « modèle munichois »

Des programmes spécifiques également élaborés à Hambourg...

... et à Berlin 
Stratégies alternatives de prolongation des périodes de subventionnement

Une solution pour les personnes privées de logement personnel ?

La construction de logements pour les classes moyennes pourrait venir désengorger le marché

Inconvénients du soutien apporté à l'offre de logements sociaux

L'aide aux locataires : une réponse mieux ciblée
Si la construction de logements sociaux s'avère souvent rentable pour les investisseurs, dans la concurrence en matière de terrains en revanche, le marché libre de la construction de logements s'impose dans les zones attractives et moyennement attractives, car il reste nettement plus lucratif (Faller, 2012).

Face à cet état de fait, une solution complémentaire peut consister pour les villes à prolonger la phase de subventionnement lorsque celle-ci prend fin et que le locataire n'a pas les moyens de verser un loyer tel que ceux appliqués à l'échelon local. Potsdam souhaite ainsi attribuer, en moyenne annuelle, non moins de 120 logements à des ménages à faibles revenus.

Ces politiques pourraient venir enrayer la hausse $(+15 \%$ depuis 2010$) d u$ nombre de personnes privées de logement personnel, c'est-à-dire vivant pour la plupart chez des proches ou dans des centres d'accueil, désormais estimé à près de 284000 . Selon le directeur de la Bundesarbeitsgemeinschaft Wohnungslosenhilfe (BAGW), Thomas Specht, cette brusque augmentation serait étroitement liée à la hausse des prix des loyers dans les agglomérations. Le nombre des sans-abri a pour sa part augmenté de $10 \%$ en 2011 et 2012, atteignant aujourd'hui près de 24000 personnes.

Selon l'étude de l'entreprise de conseil RegioKontext évoquée plus haut, le développement du logement social doit être complété par un soutien à la construction de logements pour les classes moyennes. En effet, la construction de nouveaux logements sur le segment de milieu de gamme réduira non seulement les tensions sur le marché du logement dans les grandes villes, mais contribuera également, directement ou indirectement, à améliorer la situation sur le segment des habitations à loyer modéré. En effet, les ménages appartenant aux classes moyennes libéreront des logements à loyer modéré qui par exemple ne répondront plus à leurs attentes (effet direct) ou ils ne chercheront plus de logement sur le segment des prix moyens et modérés (effet indirect) car l'offre correspondra davantage à la demande.

Les chercheurs de l'IW relèvent pour leur part un conflit d'intérêts entre le fait de souhaiter viser les populations les plus défavorisées et de favoriser la mixité sociale. Par ailleurs, le fait que les personnes dont les revenus augmentent restent dans les logements sociaux entraîne un problème en termes de ciblage des populations. Enfin, ils considèrent cet instrument comme peu flexible car, en raison du vieillissement démographique, la situation va s'améliorer dans de nombreuses régions qui connaissent actuellement une pénurie, et ce en raison d'un recul de la demande à partir de 2030. Or les logements sociaux seront alors toujours disponibles, voire parfois subventionnés.

\section{Aides aux locataires}

Pour ces mêmes spécialistes du marché du logement, l'augmentation de l'allocation logement (Wohngeld) pour les personnes aux revenus faibles et moyens serait plus efficace que la construction de nouveaux logements sociaux, car ce dispositif viserait plus précisément les ménages à faibles revenus, au moment où ils en ont besoin. Début 2013, le ministre fédéral des Transports, de la Construction et de l'Urbanisme Peter Ramsauer (CSU) s'est dit disposé à aborder un relèvement du plafond relatif au Wohngeld.

\section{Allocation logement (Wohngeld)}

et prise en charge des frais de loyer et de chauffage $(K d U)$

L'allocation logement (Wohngeld), qui constitue une aide partielle aux coûts du logement pour les personnes à faibles revenus, diffère du remboursement complet des frais de loyer et de chauffage (Kosten der Unterkunft und Heizung, $K d U)$, effectué dans le cadre de la garantie du revenu minimum de subsistance et destiné aux allocataires de prestations sociales. Le nombre de bénéficiaires du Wohngeld a fortement reculé depuis la réforme Hartz IV de 2005, ces derniers ayant souvent basculé vers le régime $K d U$.

Un montant à réévaluer en tenant compte des évolutions du marché
En 2010, l'allocation logement concernait 852400 foyers (soit 2,1\% des ménages allemands), qui percevaient $126 €$ en moyenne. L'Etat fédéral et les Län- 
der avaient alors dépensé 1,78 milliard $€$, soit deux fois plus qu'en 2008. La même année, la prise en charge des frais de loyer et de chauffage représentait un budget de 15,2 milliards $€$. Le montant de cette aide, mesuré par rapport aux loyers pratiqués dans la zone géographique considérée, doit être parfaitement adapté. S'il était trop élevé en effet, il pourrait générer une augmentation des loyers, au détriment des ménages peu aisés qui ne perçoivent pas cette aide. Dans le cas où il serait trop faible, cela rendrait l'accès au logement plus difficile pour les allocataires et pourrait aboutir à une ségrégation géographique accrue des ménages. Avec l'allocation logement et la prise en charge des frais de loyer et de chauffage, l'Etat allemand soutient cinq millions de foyers, à l'aide d'un montant total annuel de 17 milliards $€$. Un cinquième des logements locatifs en Allemagne est occupé par des ménages aidés via le Wohngeld ou le KdU (BMVBS, 2013). Comme pour le $K d U$, l'allocation logement est trop rarement réévaluée en fonction des évolutions du marché de l'immobilier. Son montant a été augmenté pour la dernière fois en 2009, tandis qu'en 2011, il a même été réduit avec la suppression du forfait chauffage.

En supplément de ces aides, le ministre fédéral des Transports, de la Construction et de l'Urbanisme, Peter Ramsauer (CSU), s'est déclaré prêt à discuter, après les élections, de la réintroduction de la prime d'accès à la propriété (Eigenheimzulage) pour les familles avec enfants.

\section{Réponses à la pénurie de terrains}

Une solution complémentaire à l'augmentation actuelle du prix des loyers pourrait résider dans l'optimisation des terrains existants, en vue d'accroître l'offre de logements, et notamment dans l'utilisation des friches industrielles résultant de la mutation structurelle de l'économie dans les grandes villes et du passage à une société de services. II pourrait s'agir également de procéder à une densification des villes, via la surélévation de bâtiments existants ou un recours accru à l'aménagement de combles.

Les bureaux vacants pourraient également être convertis en logements. En effet, à Berlin, Düsseldorf, Francfort, Hambourg, Cologne, Munich et Stuttgart, le taux moyen de bureaux vacants a passé la barre des $10 \%$ à la fin de l'année 2010. La ville de Francfort présentait le taux le plus élevé, avec 18,5\%, suivie par Düsseldorf, avec $12 \%$ (Clamor et al., 2011). Pourrait ainsi émerger dans les zones situées près des centres-villes une nouvelle offre de logements, sous réserve que ces surfaces soient bel et bien utilisables. Quoi qu'il en soit, cette solution ne peut s'envisager pour pallier un problème transitoire tel que l'accroissement actuel du nombre d'étudiants dans les villes universitaires. Le passage de 13 à 12 ans de scolarité jusqu'à l'Abitur, l'équivalent du baccalauréat en Allemagne, vient ainsi doubler sur une année les effectifs des diplômés du secondaire. Or la conversion de bureaux en espaces d'habitation s'inscrit plutôt dans un long processus d'aménagement et de construction.

Malgré tout, les villes doivent faire face à une pénurie de terrains. A Munich par exemple, 80000 logements de plus seraient nécessaires d'ici 2030, mais les réserves de terrains ne permettent la construction que de 50000 logements. Les Munichois se prononcent contre l'accroissement de la densité démographique, tandis que les maires des petites villes voisines souhaitent conserver des structures villageoises. En outre, avant de pouvoir accueillir une nouvelle population de parents actifs, les communes environnantes devraient développer les structures d'accueil des jeunes enfants. Or de nombreuses villes sont réservées sur ce point en raison du coût que cela induit. Se pose également la question des infrastructures de transport et de la fréquence des liaisons proposées, et ce alors même qu'une installation à la périphérie des villes ne semble plus présenter d'intérêt financier particulier pour les ménages : les loyers y sont déjà élevés, tandis que les coûts liés à la mobilité viennent (au mieux) compenser le gain réalisé sur le coût du logement.
Vers une réintroduction de la prime d'accès à la propriété ?

Utilisation des friches industrielles et densification urbaine

Conversion de bureaux en espaces d'habitation

Quels potentiels en périphérie des villes? 
FACE À L'AUgmentATION DU PRIX DES LOYERS dans les grandes villes allemandes, la question du logement, étroitement liée à la protection sociale, à la transition énergétique et à la stabilité des marchés financiers, revient au cœur des débats, l'objectif ultime étant de préserver la mixité sociale dans les villes. Or la construction de logements est un processus faisant intervenir de nombreux acteurs : un consensus politique entre communes, Länder et Etat fédéral est nécessaire pour établir un cadre favorable à la construction, qui reste toutefois du ressort du secteur de l'immobilier et des maîtres d'ouvrages privés. En se concentrant sur les quelques villes où la demande augmente, les responsables politiques occultent le problème du recul de la population observable dans les régions et villes allemandes économiquement faibles où le taux de logement vacants est élevé, et ce d'autant que le problème observé actuellement dans les villes allemandes est transitoire : à partir de 2030, la demande reculera également dans les grandes villes en raison du vieillissement démographique.

\section{Indications bibliographiques}

-ANKENBRAND H., KLoepfer I., « Der neue Häuserkampf », Frankfurter Allgemeine Zeitung [FAZ], 20/01/2013

-BRAUn R., « CBRE-empirica-Leerstandsindex : marktaktive Quote im 5. Jahr rückläufig », Empirica Paper, $\mathrm{n}^{\circ} 213$, mai 2013

- Bundesministerium für Verkehr, Bau Und Stadtentwicklung [BMVBS], Bericht über die Wohnungs- und Immobilienwirtschaft in Deutschland, BMVBS, Berlin, 2013

- Clamor T., HAAS H., Voigtländer M., «Büroleerstand - ein zunehmendes Problem des deutschen Immobilienmarktes", IW-Trends - Vierteljahresschrift zur empirischen Wirtschaftsforschung aus dem Institut der deutschen Wirtschaft Köln, vol. 38, n 4, 2011

- DorfFMeISTeR L., GluCh E., Entwicklung des Wohnungsbaus in Deutschland bis 2016 - eine Studie des Ifo-Instituts für die Wüstenrotstiftung, Wüstenrot Bausparkasse AG, Munich, 2013

- Faller B., "Strategien für eine soziale Ausrichtung des Wohnungsmarktes », in BundesminISTERIUM FÜR VERKEHR, BAU UND STADTENTWICKLUNG, Werkstattgespräch: „Wohnungspolitik in Wachstumsregionen", BMVBS, Berlin, 2012

- GePPERT K., GorNig M., « Mehr Jobs, mehr Menschen : Die Anziehungskraft der großen Städte wächst », Wochenbericht des DIW Berlin, n 19, 2010

- HAAs H., Henger R., Voigtländer M., "Reale Nachfrage oder bloße Spekulation - Ist der deutsche Wohnimmobilienmarkt überhitzt? », IW policy paper, $\mathrm{n}^{\circ}$ 8, 2013

- HAZOUARD S. «BTP allemand : restructuration et nouveaux enjeux », Regards sur l'économie allemande, $\mathrm{n}^{\circ} 102,2011$

- INSTITUT DER DEUTSCHEN WIRTSChAFT (ed.), Immobilienpolitik 2013 - Die Wahlprogramme von Union, FDP, SPD und Grüne im Vergleich - Gutachten für den ZIA Zentraler Immobilienausschuss e.V., IW Köln, Cologne, 2013

- Institut für Demoskopie Allensbach, «Die aktuelle Situation auf dem Wohnungsmarkt aus Sicht der Bevölkerung - Hohe Mietpreise und Wohnraumknappheit sind vor allem ein Problem der Großstädte », Allensbacher Kurzbericht, n² 4, août 2013

- Pestel InStITUt, Bedarf an Sozialwohnungen in Deutschland, Pestel Institut, Hanovre, 2012

- REgIOKONTEXT, Strategien für bezahlbares Wohnen in der Stadt - Welchen Beitrag kann der Neubau angesichts neuer Wohnungsknappheit leisten?, RegioKontext $\mathrm{GmbH}$, Berlin, 2013

- SPARS G., Heinze M., « Optionen für bezahlbaren Wohnraum in den Großstädten », Forum Wohnen und Stadtentwicklung, $\mathrm{n}^{\circ}$ 3, mai-juin 2013

- SPARS G., «Aktuelle Entwicklung der Wachstumsregionen », in BUNDESMinISTERIUm für Verkehr, BAU UND STADTENTWICKLUNG, Werkstattgespräch: "Wohnungspolitik in Wachstumsregionen", BMVBS, Berlin, 2012

-http://ec.europa.eu/eurostat

- http://www.bmvbs.de

$\bullet$ http://www.destatis.de. 